\title{
Association study between autistic-like traits and polymorphisms in the autism candidate regions RELN, CNTNAP2, SHANK3, and CDH9/10
}

Lina Jonsson ${ }^{1 *}$, Anna Zettergren ${ }^{1}$, Erik Pettersson ${ }^{2}$, Daniel Hovey ${ }^{1}$, Henrik Anckarsäter ${ }^{3}$, Lars Westberg ${ }^{1}$, Paul Lichtenstein ${ }^{2}$, Sebastian Lundström ${ }^{3,4,5}$ and Jonas Melke ${ }^{1}$

\begin{abstract}
Background: Autistic-like traits (ALTs) are continuously distributed in the general population, with the autism spectrum disorder (ASD) at the upper extreme end. A genetic overlap has been shown between ALTs and ASD, indicating that common variation in ASD candidate genes may also influence ALTs. In our study, we have investigated the SNP rs4307059 that has been associated with both ALTs and ASD. In addition, we genotyped polymorphisms in a selection of genes involved in synaptic functioning, that is, SHANK3, RELN, and CNTNAP2, which repeatedly have been associated with ASD. The possible associations of these polymorphisms with ALTs, as well as genetic factors for neurodevelopmental problems (NDPs), were investigated in a large cohort from the general population: The Child and Adolescent Twin Study in Sweden. For analyses of ALTs and NDPs, 12,319 subjects (including 2,268 monozygotic (MZ) and 3,805 dizygotic (DZ) twin pairs) and 8,671 subjects (including 2,243 MZ and 2,044 DZ twin pairs), respectively, were included in the analyses.
\end{abstract}

Findings: We could not replicate the previous association between rs4307059 and social communication impairment. Moreover, common variations in CNTNAP2 (rs7794745 and rs2710102), RELN (rs362691), and SHANK3 (rs9616915) were not significantly associated with ALTs in our study.

Conclusions: Our results do not suggest that the investigated genes, which previously has been found associated with ASD diagnosis, have any major influence on ALTs in children from the general population.

Keywords: Autistic-like traits, Autism spectrum disorder, CNTNAP2, RELN, rs4307059, SHANK3, A-TAC, CATSS

\section{Findings}

\section{Background}

Autism spectrum disorder (ASD) is a heterogeneous group of neurodevelopmental disorders that are characterized by impairments in social interaction restricted and repetitive behavior and communication impairments. Furthermore, there is a large co-morbidity between ASD and other neurodevelopmental disorders, such as Attention Deficit/Hyperactive Disorder (ADHD) [1] and a shared genetic susceptibility has been suggested for neurodevelopmental disorders [2].

\footnotetext{
* Correspondence: lina.jonsson@neuro.gu.se

'Department of Pharmacology, Institute of Neuroscience and Physiology at the Sahlgrenska Academy, University of Gothenburg, POB 431, SE 405 30,

Gothenburg, Sweden

Full list of author information is available at the end of the article
}

The phenotypic heterogeneity within ASD is probably the major explanation for the inconclusive results from genetic studies of ASD. Indeed, the majority of genes and biological pathways clearly implicated in ASD have been identified in studies of rare variation [3], whereas studies of common polymorphisms, both in large, genome-wide association studies (GWAS) [4-6] and smaller hypothesis driven studies have yielded inconclusive results [3]. Another approach to investigate the role of common variants in ASD is to assess milder, non-pathological phenotypes related to ASD, such as autistic-like traits (ALTs), that are dimensionally distributed in the general population [7]. Theoretically, common variations would have a larger impact

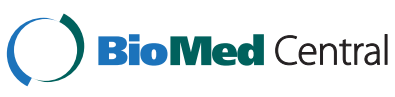

(c) 2014 Jonsson et al.; licensee BioMed Central Ltd. This is an Open Access article distributed under the terms of the Creative Commons Attribution License (http://creativecommons.org/licenses/by/4.0), which permits unrestricted use, distribution, and reproduction in any medium, provided the original work is properly credited. The Creative Commons Public Domain Dedication waiver (http://creativecommons.org/publicdomain/zero/1.0/) applies to the data made available in this article, unless otherwise stated. 
for ALTs than for severe ASD [3], and this approach also allows for analyses of the genetics for different aspects of the autistic phenotype separately [8]. Indeed, it has been shown that ALTs and clinical ASD are etiologically related [7] and one example of a single nucleotide polymorphism (SNP) that has been associated both with ASD [5] and social interaction impairment is a SNP (rs4307059) between the genes Cadherin 9 and 10 (CDH9 and CDH1O) [9]. Also, a SNP (rs2710102) in the Contactin associated protein-like 2 (CNTNAP2) has been shown to affect language development in the general population [10] and age at first word in children with ASD [11].

Genetically determined abnormalities in neurodevelopment and synaptic functioning are increasingly recognized as a cause of ASD. Although these findings primarily are based on rare mutations identified in ASD families [12-14], there is evidence for an influence of also common polymorphisms in ASD [3]. In the present study we initially evaluated the association between common variation in three genes involved in neurodevelopment and/or synaptic function, that is, CNTNAP2, SH3, and multiple ankyrin repeat domains 3 (SHANK3) and Reelin $(R E L N)$, and continuous measures of ALTs. Second, we investigated the possible influence of rs4307059 (between CDH9 and CDH10) and rs2710102 (CNTNAP2) on both ALTs and ASD. Finally, we explored the influence of the five SNPs on neurodevelopmental problems (NDPs). Thus, the purpose of our study was to expand and partly replicate previous findings regarding these SNPs in autism related phenotypes.

\section{Subjects and methods}

\section{Participants and measurements}

The subjects in our study are part of The Child and Adolescent Twin Study in Sweden (CATSS) that is focusing on neurodevelopmental problems in children [15]. Our sample from the CATSS included 2,301 monozygotic (MZ) twin pairs, 3,870 dizygotic (DZ) twin pairs, and 84 subjects included without their co-twin. From this sample, we have excluded 107 subjects due to documented brain damage or a known genetic syndrome; a total of 12,319 subjects were included in our analyses of ALTs. For analysis of NDPs a total of 8,671 subjects were included: 2,243 MZ and 2,044 DZ twin pairs, and 97 subjects without their co-twin. The CATSS study has ethical approval from the Karolinska Institutet Ethical Review Board, and informed consent was obtained from the participants.

The parental questionnaire Autism-Tics, ADHD, and other Co-morbidities inventory (A-TAC) was answered by the parents in connection to their twins' 9th or 12th birthday [16,17]; $70 \%$ are 9 years old $(\mathrm{N}=8,623)$ and $30 \%$ are 12 years old $(\mathrm{N}=3,696)$ at the time of testing. The A-TAC consists of 96 questions, of which 17 measure
ALTs: six questions correspond to the language impairment, six to the social interaction impairment, and five to restricted and repetitive behavior. The A-TAC score can also be used as a proxy for a clinical ASD diagnosis if the children score higher than 8.5 points on the scale $(\mathrm{N}=90$ in our sample) [17]. Furthermore, 53 items in the A-TAC have been used to identify a general genetic factor, that is, a factor that consists of all neurodevelopmental problems (NDPs) as well as three genetic subfactors (tics and autism, hyperactivity, and learning problems) that were independent of the general genetic factor and primarily identified by a smaller number of specific symptoms [18]. These four factors were based on over 6,500 twin pairs and were designed to have $100 \%$ heritability by fixing the correlations across twin pairs at their expected genetic pedigree (that is, at $r=1.0$ for $\mathrm{MZ}$ and at $\mathrm{r}=0.50$ for $\mathrm{DZ}$ twins). Since the calculations are based on a heritability estimate that is twice the difference between $\mathrm{MZ}$ and $\mathrm{DZ}$ twins, the factors end up having $100 \%$ heritability. Please note that the $100 \%$ heritability is a statistical routine to maximize factor loading, rather than an actual estimate of the heritability of these traits. By relying on the expected twin correlations, factor analysis identified the specific set of weights for the items that were perfectly heritable. Because one factor had substantial and positive weights on all 53 items, it was considered general, that is, all symptoms partly shared the same genetic origin. Given that these factors were entirely genetic in origin, their associations with specific candidate genes may emerge more clearly.

\section{Genotyping and statistical analyses}

Five single nucleotide polymorphisms (SNPs) in the genes CNTNAP2 (rs2710102 and rs7794745), RELN (rs362691), SHANK3 (rs9616915), and rs4307059 between $\mathrm{CDH} 9$ and $\mathrm{CDH} 10$ were genotyped. DNA was extracted from saliva using OraGene DNA Self-collection Kit (DNA Genotek Inc., Ottawa, Canada) and the SNPs were genotyped using the Kompetitive Allele Specific PCR (KASP) Genotyping System (LGC Genomics, Herts, UK). All of the SNPs were in Hardy-Weinberg Equilibrium $(P>0.01)$ and the genotyping success rate was above $97 \%$.

Statistical association, for ALTs and NDPs, were estimated using linear mixed effect models in the MIXED procedure (PROC MIXED) and for the case-control analyses we used the GLIMMIX procedure of SAS 9.3 (SAS Institute, Inc., Cary, NC, USA). Both of these models allowed us to adjust for the dependent nature of the twin observations, that is, A-TAC scores from all genotyped subjects were included in the analyses. For the MIXED procedure, the genotypes were coded as quantitative measures $(0=$ major allele homozygote, $1=$ heterozygote, and 2 = minor allele homozygote) and effect sizes are presented as the regression coefficients for these 
Table 1 Association between five SNPs and autistic-like traits

\begin{tabular}{|c|c|c|c|c|c|c|c|c|c|c|c|c|c|c|c|c|c|}
\hline \multirow[b]{3}{*}{$\begin{array}{l}\text { Gene/ } \\
\text { SNP }\end{array}$} & \multirow[b]{3}{*}{ MAF } & \multicolumn{7}{|c|}{ Autistic-like traits $^{\mathbf{a}}$} & \multicolumn{9}{|c|}{ Autistic-like traits domains } \\
\hline & & \multirow[b]{2}{*}{ Genotype } & \multirow[b]{2}{*}{$N$} & \multirow[b]{2}{*}{ All } & \multirow[b]{2}{*}{$\mathbf{N}$} & \multirow[b]{2}{*}{ Boys } & \multirow[b]{2}{*}{$\mathrm{N}$} & \multirow[b]{2}{*}{ Girls } & \multicolumn{3}{|c|}{ Restricted \& repetitive behavior ${ }^{\mathrm{a}}$} & \multicolumn{3}{|c|}{ Social interaction impairment ${ }^{\mathrm{a}}$} & \multicolumn{3}{|c|}{ Language impairment $^{\mathrm{a}}$} \\
\hline & & & & & & & & & All & Boys & Girls & All & Boys & Girls & All & Boys & Girls \\
\hline \multicolumn{18}{|l|}{$\mathrm{CDH} 9 / 10$} \\
\hline \multirow[t]{3}{*}{ rs4307059 } & $\begin{array}{l}0.37 \\
\text { (C) }\end{array}$ & $\mathrm{C} / \mathrm{C}$ & 1,663 & $0.68(1.37)$ & 796 & $0.86(1.63)$ & 867 & $0.50(1.06)$ & $0.23(0.57)$ & $0.31(0.69)$ & $0.16(0.42)$ & $0.23(0.57)$ & $0.29(0.64)$ & $0.18(0.49)$ & $0.21(0.52)$ & $0.27(0.59)$ & $0.16(0.43)$ \\
\hline & & $\mathrm{T} / \mathrm{C}$ & 5,564 & $0.73(1.44)$ & 2,820 & $0.91(1.66)$ & 2,744 & $0.54(1.14)$ & $0.23(0.57)$ & $0.30(0.66)$ & $0.16(0.45)$ & $0.25(0.58)$ & $0.30(0.64)$ & $0.21(0.50)$ & $0.24(0.57)$ & $0.30(0.65)$ & $0.18(0.46)$ \\
\hline & & $T / T$ & 4,766 & $0.67(1.36)$ & 2,391 & $0.85(1.64)$ & 2,375 & $0.50(0.98)$ & $0.21(0.55)$ & $0.28(0.65)$ & $0.14(0.40)$ & $0.24(0.58)$ & $0.29(0.68)$ & $0.19(0.45)$ & $0.22(0.54)$ & $0.27(0.62)$ & $0.17(0.44)$ \\
\hline \multicolumn{2}{|l|}{$P$ value } & & & 0.737 & & 0.634 & & 0.724 & 0.160 & 0.302 & 0.162 & 0.793 & 0.762 & 0.669 & 0.725 & 0.704 & 0.357 \\
\hline \multicolumn{3}{|c|}{ Effect size $\left(\mathrm{Cl}\right.$ 95\%) ${ }^{\mathrm{C}}$} & & $\begin{array}{l}0.007 \\
(-0.033 \\
0.047)\end{array}$ & & $\begin{array}{l}0.016 \\
(-0.051 \\
0.083)\end{array}$ & & $\begin{array}{l}0.007 \\
(-0.033 \\
0.048)\end{array}$ & $\begin{array}{l}0.011 \\
(-0.004 \\
0.027)\end{array}$ & $\begin{array}{l}0.014 \\
(-0.013 \\
0.041)\end{array}$ & $\begin{array}{l}0.011 \\
(-0.005 \\
0.028)\end{array}$ & $\begin{array}{l}-0.002 \\
(-0.018 \\
0.014)\end{array}$ & $\begin{array}{l}-0.004 \\
(-0.03, \\
0.022)\end{array}$ & $\begin{array}{l}0.004 \\
(-0.014 \\
0.022)\end{array}$ & $\begin{array}{l}0.003 \\
(-0.019 \\
0.013)\end{array}$ & $\begin{array}{l}0.005 \\
(-0.020 \\
0.030)\end{array}$ & $\begin{array}{l}-0.008 \\
(-0.026 \\
0.009)\end{array}$ \\
\hline \multicolumn{18}{|l|}{ CNTNAP2 } \\
\hline \multirow[t]{3}{*}{ rs2710102 } & $\begin{array}{l}0.48 \\
(\mathrm{G})\end{array}$ & $\mathrm{A} / \mathrm{A}$ & 3,248 & $0.69(1.37)$ & 1,618 & $0.87(1.63)$ & 1,630 & $0.52(1.03)$ & $0.22(0.57)$ & $0.29(0.68)$ & $0.15(0.43)$ & $0.25(0.57)$ & $0.30(0.65)$ & $0.20(0.48)$ & $0.22(0.53)$ & $0.27(0.61)$ & $0.17(0.42)$ \\
\hline & & $A / G$ & 5,976 & $0.70(1.41)$ & 2,998 & $0.88(1.68)$ & 2,978 & $0.52(1.04)$ & $0.22(0.56)$ & $0.29(0.66)$ & $0.15(0.42)$ & $0.24(0.58)$ & $0.29(0.66)$ & $0.20(0.47)$ & $0.23(0.57)$ & $0.29(0.65)$ & $0.18(0.46)$ \\
\hline & & $\mathrm{G} / \mathrm{G}$ & 2,752 & $0.73(1.45)$ & 1,382 & $0.90(1.60)$ & 1,370 & $0.55(1.24)$ & $0.23(0.58)$ & $0.31(0.65)$ & $0.16(0.47)$ & $0.25(0.58)$ & $0.29(0.64)$ & $0.21(0.51)$ & $0.24(0.56)$ & $0.30(0.62)$ & $0.18(0.49)$ \\
\hline \multicolumn{3}{|l|}{$P$ value } & & 0.523 & & 0.652 & & 0.727 & 0.593 & 0.566 & 0.880 & 0.827 & 0.653 & 0.766 & 0.336 & 0.368 & 0.542 \\
\hline \multicolumn{3}{|c|}{ Effect size $(\mathrm{Cl} 95 \%)^{c}$} & & $\begin{array}{l}0.013 \\
(-0.026 \\
0.052)\end{array}$ & & $\begin{array}{l}0.015 \\
(-0.050 \\
0.080)\end{array}$ & & $\begin{array}{l}0.007 \\
(-0.033 \\
0.048)\end{array}$ & $\begin{array}{l}0.004 \\
(-0.011 \\
0.020)\end{array}$ & $\begin{array}{l}0.008 \\
(-0.018 \\
0.033)\end{array}$ & $\begin{array}{l}-0.001 \\
(-0.017 \\
0.015)\end{array}$ & $\begin{array}{l}-0.002 \\
(-0.018 \\
0.014)\end{array}$ & $\begin{array}{l}-0.006 \\
(-0.031 \\
0.020)\end{array}$ & $\begin{array}{l}0.003 \\
(-0.015 \\
0.020)\end{array}$ & $\begin{array}{l}0.008 \\
(-0.008, \\
0.023)\end{array}$ & $\begin{array}{l}0.011 \\
(-0.013 \\
0.036)\end{array}$ & $\begin{array}{l}0.006 \\
(-0.012 \\
0.023)\end{array}$ \\
\hline \multirow[t]{3}{*}{ rs7794745 } & $\begin{array}{l}0.36 \\
(T)\end{array}$ & $\mathrm{A} / \mathrm{A}$ & 5,004 & $0.72(1.46)$ & 2,475 & $0.92(1.73)$ & 2,529 & $0.53(1.10)$ & $0.24(0.60)$ & $0.32(0.71)$ & $0.16(0.44)$ & $0.25(0.59)$ & $0.30(0.67)$ & $0.19(0.49)$ & $0.24(0.57)$ & $0.29(0.65)$ & $0.19(0.47)$ \\
\hline & & T/A & 5,533 & $0.70(1.41)$ & 2,807 & $0.87(1.63)$ & 2,726 & $0.53(1.13)$ & $0.22(0.55)$ & $0.28(0.63)$ & $0.15(0.44)$ & $0.26(0.59)$ & $0.30(0.66)$ & $0.21(0.50)$ & $0.23(0.56)$ & $0.29(0.64)$ & $0.17(0.46)$ \\
\hline & & $T / T$ & 1,564 & $0.67(1.23)$ & 779 & $0.82(1.44)$ & 785 & $0.51(0.97)$ & $0.22(0.52)$ & $0.29(0.59)$ & $0.15(0.42)$ & $0.22(0.50)$ & $0.25(0.58)$ & $0.20(0.42)$ & $0.22(0.51)$ & $0.28(0.59)$ & $0.16(0.41)$ \\
\hline \multicolumn{3}{|l|}{$P$ value } & & 0.102 & & $0.045^{\mathrm{b}}$ & & 0.744 & 0.126 & 0.081 & 0.847 & 0.309 & $0.050^{\mathrm{b}}$ & 0.311 & 0.182 & 0.299 & 0.480 \\
\hline \multicolumn{3}{|c|}{ Effect size $(\mathrm{Cl} 95 \%)^{\mathrm{C}}$} & & $\begin{array}{l}-0.034 \\
(-0.075 \\
0.007)\end{array}$ & & $\begin{array}{l}-0.069 \\
(-0.136 \\
-0.001)\end{array}$ & & $\begin{array}{l}0.007 \\
(-0.035 \\
0.049)\end{array}$ & $\begin{array}{l}-0.013 \\
(-0.029 \\
0.004)\end{array}$ & $\begin{array}{l}-0.024 \\
(-0.051 \\
0.003)\end{array}$ & $\begin{array}{l}-0.002 \\
(-0.018 \\
0.015)\end{array}$ & $\begin{array}{l}-0.009 \\
(-0.025 \\
0.008)\end{array}$ & $\begin{array}{l}-0.026 \\
(-0.053 \\
<0.001)\end{array}$ & $\begin{array}{l}0.009 \\
(-0.009, \\
0.028)\end{array}$ & $\begin{array}{l}-0.011 \\
(-0.027 \\
0.005)\end{array}$ & $\begin{array}{l}-0.014 \\
(-0.039 \\
0.012)\end{array}$ & $\begin{array}{l}-0.007 \\
(-0.025 \\
0.012)\end{array}$ \\
\hline \multicolumn{18}{|l|}{ RELN } \\
\hline \multirow[t]{3}{*}{ rs362691 } & $\begin{array}{l}0.13 \\
\text { (C) }\end{array}$ & $\mathrm{C} / \mathrm{C}$ & 211 & $0.65(1.34)$ & 107 & $0.66(1.11)$ & 104 & $0.63(1.54)$ & $0.22(0.56)$ & $0.25(0.54)$ & $0.18(0.58)$ & $0.25(0.55)$ & $0.24(0.44)$ & $0.25(0.65)$ & $0.18(0.45)$ & $0.17(0.41)$ & $0.20(0.49)$ \\
\hline & & $C / G$ & 2,629 & $0.68(1.39)$ & 1,267 & $0.89(1.69)$ & 1,362 & $0.49(1.01)$ & $0.21(0.56)$ & $0.30(0.68)$ & $0.14(0.40)$ & $0.24(0.60)$ & $0.31(0.71)$ & $0.18(0.46)$ & $0.22(0.54)$ & $0.29(0.61)$ & $0.16(0.46)$ \\
\hline & & $\mathrm{G} / \mathrm{G}$ & 9,139 & $0.71(1.40)$ & 4,615 & $0.88(1.63)$ & 4,524 & $0.53(1.09)$ & $0.22(0.56)$ & $0.29(0.66)$ & $0.15(0.43)$ & $0.25(0.57)$ & $0.29(0.64)$ & $0.20(0.48)$ & $0.23(0.56)$ & $0.29(0.64)$ & $0.18(0.45)$ \\
\hline$P$ value & & & & 0.338 & & 0.629 & & 0.659 & 0.350 & 0.739 & 0.612 & 0.944 & 0.835 & 0.980 & 0.282 & 0.306 & 0.659 \\
\hline Effect size & (Cl 95\%) & & & $\begin{array}{l}-0.028 \\
(-0.086 \\
0.03)\end{array}$ & & $\begin{array}{l}-0.024 \\
(-0.12 \\
0.072)\end{array}$ & & $\begin{array}{l}-0.013 \\
(-0.073 \\
0.046)\end{array}$ & $\begin{array}{l}-0.011 \\
(-0.034 \\
0.012)\end{array}$ & $\begin{array}{l}-0.007 \\
(-0.045 \\
0.032)\end{array}$ & $\begin{array}{l}-0.006 \\
(-0.03 \\
0.017)\end{array}$ & $\begin{array}{l}-0.001 \\
(-0.024 \\
0.022)\end{array}$ & $\begin{array}{l}0.004 \\
(-0.034 \\
0.042)\end{array}$ & $\begin{array}{l}<0.001 \\
(-0.026 \\
0.026)\end{array}$ & $\begin{array}{l}-0.013 \\
(-0.036 \\
0.010)\end{array}$ & $\begin{array}{l}-0.019 \\
(-0.056 \\
0.018)\end{array}$ & $\begin{array}{l}-0.006 \\
(-0.032 \\
0.020)\end{array}$ \\
\hline
\end{tabular}


Table 1 Association between five SNPs and autistic-like traits (Continued)

\begin{tabular}{|c|c|c|c|c|c|c|c|c|c|c|c|c|c|c|c|c|c|}
\hline \multirow[t]{3}{*}{ rs9616915 } & $\begin{array}{l}0.49 \\
(T)\end{array}$ & $\mathrm{C} / \mathrm{C}$ & 3,116 & $0.74(1.53)$ & 1,589 & $0.96(1.84)$ & 1,527 & $0.52(1.07)$ & $0.23(0.60)$ & $0.32(0.72)$ & $0.14(0.43)$ & $0.27(0.62)$ & $0.33(0.73)$ & $0.20(0.48)$ & $0.24(0.58)$ & $0.31(0.68)$ & $0.18(0.44)$ \\
\hline & & $C / T$ & 5,839 & $0.68(1.32)$ & 2,906 & $0.83(1.51)$ & 2,933 & $0.53(1.08)$ & $0.22(0.56)$ & $0.29(0.65)$ & $0.16(0.44)$ & $0.24(0.54)$ & $0.28(0.60)$ & $0.20(0.47)$ & $0.22(0.52)$ & $0.26(0.58)$ & $0.18(0.45)$ \\
\hline & & $\mathrm{T} / \mathrm{T}$ & 2,927 & $0.68(1.38)$ & 1,461 & $0.88(1.62)$ & 1,466 & $0.49(1.04)$ & $0.21(0.53)$ & $0.29(0.62)$ & $0.14(0.41)$ & $0.24(0.56)$ & $0.28(0.64)$ & $0.19(0.48)$ & $0.24(0.57)$ & $0.31(0.67)$ & $0.16(0.45)$ \\
\hline \multicolumn{3}{|l|}{$P$ value } & & 0.194 & & 0.425 & & 0.445 & 0.182 & 0.274 & 0.662 & 0.164 & 0.184 & 0.778 & 0.840 & 0.637 & 0.438 \\
\hline \multicolumn{3}{|c|}{ Effect size $(\mathrm{Cl} 95 \%)^{\mathrm{C}}$} & & $\begin{array}{l}-0.026 \\
(-0.064 \\
0.013)\end{array}$ & & $\begin{array}{l}-0.026 \\
(-0.089 \\
0.038)\end{array}$ & & $\begin{array}{l}-0.015 \\
(-0.055 \\
0.024)\end{array}$ & $\begin{array}{l}-0.010 \\
(-0.026 \\
0.005)\end{array}$ & $\begin{array}{l}-0.014 \\
(-0.04 \\
0.011)\end{array}$ & $\begin{array}{l}-0.004 \\
(-0.019 \\
0.012)\end{array}$ & $\begin{array}{l}-0.011 \\
(-0.027 \\
0.005)\end{array}$ & $\begin{array}{l}-0.017 \\
(-0.042, \\
0.008)\end{array}$ & $\begin{array}{l}-0.003 \\
(-0.02 \\
0.015)\end{array}$ & $\begin{array}{l}-0.002 \\
(-0.017, \\
0.014)\end{array}$ & $\begin{array}{l}0.006 \\
(-0.018 \\
0.030)\end{array}$ & $\begin{array}{l}-0.007 \\
(-0.024 \\
0.010)\end{array}$ \\
\hline
\end{tabular}

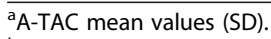

${ }^{b}$ Nominal significance, $P$ value $<0.05$

${ }^{C}$ Effect size presented as the regression coefficient (Cl 95\%)

A-TAC: Autism-Tics, ADHD, and Other Co-morbidities inventory. 
analyses. To consider the possible influence of age at testing, all analyses were also performed adjusted for age (see Additional file 1). The Bonferroni corrected $P$ value was set to $0.0016(<0.05 / 32$, adjusted for eight A-TAC measurements and four genetic regions).

We used the Quanto software to analyze which effect sizes ( $\beta$-value) we would be able to detect with a power of $90 \%$ or more using an additive genetic model at a significance level of 0.0016 [19]. The mean values and standard deviation for ALTs, ALT modules, and NDPs were used for the power analyses $(\mathrm{N}=12,319)$. For ALTs we had $90 \%$ power to detect the following effect sizes: $\beta$ value between 0.08 and 0.09 for all SNPs except for rs36269 $(\beta=0.12, \mathrm{MAF}=0.13)$. For the ALT domains (language and social impairment, and restricted and repetitive behavior) the effect size ( $\beta$-values) ranged between 0.04 and 0.05 and for the genetic factors for NDPs it ranged between 0.03 and 0.06 .

\section{Results and discussion}

Our approach was to investigate ALTs and common genetic variations previously implicated in ASD, that is, polymorphisms in CNTNAP2, SHANK3, RELN, and the SNP rs4307059 between $C D H 9$ and $C D H 10$. We could not find any significant association with ALTs for any of the investigated SNPs, however, we did see a nominal significance for the association between the CNTNAP2 SNP rs7794745 and both social interaction impairment and total ALT scores (Table 1).

In the CNTNAP2 gene, the SNP rs7794745 has been associated with autistic disorder [20] while the SNP rs2710102 has mainly been associated with language problems [11]. A study by Whitehouse et al. found nominal significance between rs2710102 (CNTNAP2) and quantitative measures of early language acquisition in the general population [10], which was not replicated in our study (Table 1).

The SNP rs4307059 in the CDH 9/10 region has previously been associated with ASD in a GWAS [5], and was replicated in a study by Ma et al. [21]. This SNP has also been associated with social communication impairments in a large population [9], however, in line with the results from the two GWAS for autistic traits [22,23], this could not be replicated in our study (Table 1). However, it should be noted that one of the ALT GWAS included a pooled SNP analysis [23] and the other had a sample size of 965 subjects [22]. The inconsistency in results may also be due to the use of different measures of ALTs in the studies. To compare our results from previous case-control studies, we used the A-TAC score as a proxy for an ASD diagnosis [17]; rs4307059 was not significantly associated in this case-control analysis (Table 2). Notably, we had low statistical power in these analyses due to the small number of children with A-TAC scores corresponding to an ASD diagnosis.

The rs362691 in RELN has been associated with ASD in a recent meta-analysis that included five association studies [24]; one of the included studies showed significant association [25]. In SHANK3, mainly rare genetic variations have been identified in subjects with ASD [14] and association analysis for rs9616915 has previously shown negative results for association with ASD in both European and Han Chinese populations [26]. The previous negative findings do not rule out a potential

Table 2 ASD $^{a}$ case-control analyses

\begin{tabular}{|c|c|c|c|c|c|}
\hline Gene & SNP & Genotype & $\mathrm{N}$ (case/control) & $P$ value $^{\mathrm{b}}$ & OR (Cl 95\%) \\
\hline \multirow[t]{3}{*}{$\mathrm{CDH} 9 / 10$} & rs4307059 & $\mathrm{C} / \mathrm{C}$ & $12 / 1,651$ & 0.835 & $1.087(0.541-2.184)$ \\
\hline & & $\mathrm{T} / \mathrm{C}$ & $37 / 5,527$ & & $1.160(0.714-1.884)$ \\
\hline & & $\mathrm{T} / \mathrm{T}$ & $36 / 4,730$ & & \\
\hline \multirow[t]{3}{*}{ CNTNAP2 } & rs2710102 & $\mathrm{A} / \mathrm{A}$ & $25 / 3,223$ & 0.800 & $0.806(0.419-1.551)$ \\
\hline & & $A / G$ & $44 / 5,932$ & & $0.846(0.468-1.529)$ \\
\hline & & $\mathrm{G} / \mathrm{G}$ & $17 / 2,735$ & & \\
\hline \multirow[t]{3}{*}{ CNTNAP2 } & rs7794745 & $A / A$ & $41 / 4,963$ & 0.125 & $0.351(0.127-0.972)$ \\
\hline & & $\mathrm{T} / \mathrm{A}$ & $40 / 5,493$ & & $0.422(0.152-1.168)$ \\
\hline & & $\mathrm{T} / \mathrm{T}$ & $5 / 1,559$ & & \\
\hline \multirow[t]{2}{*}{ RELN } & rs362691 & $C / C+C / G$ & $20 / 2,820$ & 0.933 & $1.023(0.601-1.742)$ \\
\hline & & $\mathrm{G} / \mathrm{G}$ & $63 / 9,076$ & & \\
\hline \multirow[t]{3}{*}{ SHANK3 } & rs9616915 & $\mathrm{C} / \mathrm{C}$ & $30 / 3,086$ & 0.094 & $0.764(0.425-1.373)$ \\
\hline & & $\mathrm{T} / \mathrm{C}$ & $31 / 5,808$ & & $1.371(0.769-2.447)$ \\
\hline & & $\mathrm{T} / \mathrm{T}$ & $21 / 2,906$ & & \\
\hline
\end{tabular}

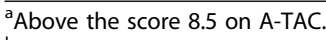

buncorrected $P$ value.

ASD: Autism spectrum disorder; A-TAC: Autism-Tics, ADHD, and Other Co-morbidities inventory. 
Table 3 Association between genetic factors for neurodevelopmental problems (NDPs) and five SNPs

\begin{tabular}{|c|c|c|c|c|c|c|c|c|c|c|c|c|c|c|c|c|c|}
\hline \multirow[b]{2}{*}{$\begin{array}{l}\text { Gene/ } \\
\text { SNP }\end{array}$} & \multirow[b]{2}{*}{ MAF } & \multirow[b]{2}{*}{ Genotype } & \multicolumn{6}{|c|}{ General NDP ${ }^{a}$} & \multicolumn{3}{|l|}{ Impulsivity $^{a}$} & \multicolumn{3}{|c|}{ Learning problems $^{a}$} & \multicolumn{3}{|c|}{ Tics and autism ${ }^{a}$} \\
\hline & & & $\mathrm{N}$ & All & $\mathbf{N}$ & Boys & $\mathrm{N}$ & Girls & All & Boys & Girls & All & Boys & Girls & All & Boys & Girls \\
\hline \multicolumn{18}{|l|}{$\overline{C D H 9 / 10}$} \\
\hline \multirow[t]{3}{*}{ rs4307059 } & $\begin{array}{l}0.37 \\
\text { (C) }\end{array}$ & $\mathrm{C} / \mathrm{C}$ & 1,149 & $0.128(0.70)$ & 538 & $0.235(0.72)$ & 611 & $0.034(0.66)$ & $0.032(0.53)$ & $0.034(0.53)$ & $0.030(0.52)$ & $0.002(0.59)$ & $0.017(0.62)$ & $\begin{array}{l}-0.010 \\
(0.57)\end{array}$ & $0.067(0.51)$ & $0.144(0.51)$ & $\begin{array}{l}-0.002 \\
(0.49)\end{array}$ \\
\hline & & $\mathrm{T} / \mathrm{C}$ & 3,923 & $0.172(0.71)$ & 1,998 & $0.275(0.73)$ & 1,925 & $0.064(0.68)$ & $0.028(0.54)$ & $0.021(0.55)$ & $0.034(0.53)$ & $0.051(0.62)$ & $0.085(0.65)$ & $0.016(0.58)$ & $0.069(0.55)$ & $0.138(0.58)$ & $\begin{array}{l}-0.003 \\
(0.50)\end{array}$ \\
\hline & & $\mathrm{T} / \mathrm{T}$ & 3,424 & $0.132(0.69)$ & 1,711 & $0.244(0.71)$ & 1,713 & $0.019(0.66)$ & $0.023(0.54)$ & $0.038(0.55)$ & $0.009(0.52)$ & $0.031(0.62)$ & $0.066(0.65)$ & $\begin{array}{l}-0.004 \\
(0.59)\end{array}$ & $0.057(0.50)$ & $0.105(0.53)$ & $0.009(0.47$ \\
\hline \multicolumn{3}{|l|}{$P$ value $^{\mathrm{b}}$} & & 0.962 & & 0.661 & & 0.811 & 0.554 & 0.571 & 0.959 & 0.401 & 0.474 & 0.702 & 0.734 & 0.702 & 0.695 \\
\hline \multicolumn{3}{|c|}{ Effect size $(C 195 \%)^{c}$} & & $\begin{array}{l}0.001 \\
(-0.021 \\
0.022)\end{array}$ & & $\begin{array}{l}0.007 \\
(-0.024 \\
0.037)\end{array}$ & & $\begin{array}{l}-0.004 \\
(-0.034 \\
0.027)\end{array}$ & $\begin{array}{l}0.005 \\
(-0.011 \\
0.021)\end{array}$ & $\begin{array}{l}0.006 \\
(-0.016 \\
0.029)\end{array}$ & $\begin{array}{l}0.001 \\
(-0.024 \\
0.025)\end{array}$ & $\begin{array}{l}-0.007 \\
(-0.023 \\
0.009)\end{array}$ & $\begin{array}{l}-0.008 \\
(-0.028 \\
0.013)\end{array}$ & $\begin{array}{l}-0.005 \\
(-0.033 \\
0.022)\end{array}$ & $\begin{array}{l}0.002 \\
(-0.012 \\
0.017)\end{array}$ & $\begin{array}{l}0.004 \\
(-0.017 \\
0.025)\end{array}$ & $\begin{array}{l}0.004 \\
(-0.018 \\
0.026)\end{array}$ \\
\hline
\end{tabular}

CNTNAP2

rs $2710102 \quad 0.48 \quad$ A/A (G)

$P$ value ${ }^{\mathrm{b}}$

Effect size $(\mathrm{Cl} 95 \%)^{c}$

rs7794745 $0.36 \quad$ A/A (T)

$P$ value ${ }^{b}$

Effect size $(\mathrm{Cl} 95 \%)^{\mathrm{C}}$

RELN

rs362691 $0.13 \quad$ C/C (C)

$2316 \quad 0.121$

.025)

.009)

013)

.022)

(-.017)

$\begin{array}{lllllllllllllll}4,242 & 0.158(0.70) & 2,126 & 0.274(0.72) & 2,116 & 0.041(0.65) & 0.030(0.54) & 0.037(0.56) & 0.023(0.53) & 0.033(0.63) & 0.075(0.67) & -0.010 \\ \end{array}$

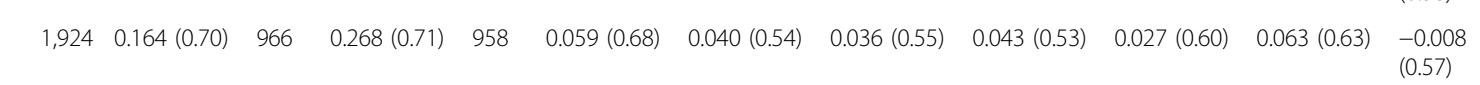

$0.057(0.53) \quad 0.126(0.56) \quad-0.012$

$0.069(0.52) \quad 0.125(0.55) \quad 0.013(0.49)$

$0.066(0.53) \quad 0.130(0.56) \quad 0.001(0.49)$

$\begin{array}{lllllllll}0.501 & 0.135 & 0.634 & 0.344 & 0.315 & 0.767 & 0.450 & 0.692 & 0.167 \\ 0.007 & 0.025 & -0.006 & 0.008 & 0.013 & 0.003 & -0.007 & 0.006 & -0.015 \\ (-0.014, & (-0.008, & (-0.032, & (-0.009, & (-0.013, & (-0.018, & (-0.025, & (-0.024, & (-0.037, \\ 0.029) & 0.057) & 0.019) & 0.025) & 0.039) & 0.025) & 0.011) & 0.037) & 0.006)\end{array}$

$\begin{array}{llllllllllllllll}3,499 & 0.144(0.70) & 1,714 & 0.251(0.72) & 1,785 & 0.041(0.67) & 0.023(0.53) & 0.028(0.54) & 0.019(0.53) & 0.022(0.61) & 0.048(0.63) & -0.004\end{array}$

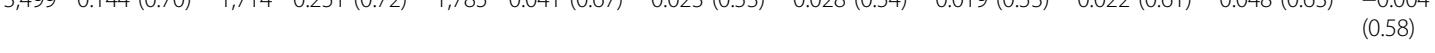

$\begin{array}{lll}0.768 & 0.856 & 0.746 \\ 0.002 & -0.002 & 0.002 \\ (-0.013, & (-0.027, & (-0.008 \\ 0.018) & 0.022) & 0.012)\end{array}$

$\begin{array}{lllllllllllllllll}3,947 & 0.156(0.70) & 2,010 & 0.269(0.71) & 1,937 & 0.039(0.67) & 0.028(0.54) & 0.035(0.56) & 0.021(0.53) & 0.046(0.62) & 0.082(0.66) & 0.009(0.58) & 0.058(0.53) & 0.116(0.56) & -0.001\end{array}$

$\begin{array}{llllllllllllllll}1,110 & 0.146(0.70) & 556 & 0.225(0.73) & 554 & 0.068(0.65) & 0.031(0.53) & 0.020(0.56) & 0.042(0.51) & 0.042(0.63) & 0.067(0.63) & 0.017(0.62) & 0.050(0.51) & 0.111(0.53) & -0.012\end{array}$

$\begin{array}{llllllllllll}0.583 & 0.169 & 0.583 & 0.590 & 0.563 & 0.905 & 0.811 & 0.547 & 0.296 & 0.112 & 0.210 & 0.567 \\ -0.006 & -0.023 & 0.007 & 0.005 & 0.007 & 0.001, & 0.002 & -0.008 & 0.012 & -0.012 & -0.015 & -0.003 \\ (-0.028, & (-0.055, & (-0.019, & (-0.012, & (-0.017, & (-0.021, & (-0.014, & (-0.032, & (-0.01, & (-0.026, & (-0.038, & (-0.013, \\ 0.016) & 0.010) & 0.034) & 0.021) & 0.031) & 0.023) & 0.018) & 0.017) & 0.034) & 0.003) & 0.008) & 0.007)\end{array}$

$\begin{array}{lllllllllllllllll}156 & 0.097(0.68) & 80 & 0.149(0.69) & 76 & 0.043(0.66) & -0.065 & -0.111 & -0.017 & 0.097(0.68) & 0.094(0.56) & 0.100(0.78) & 0.087(0.51) & 0.194(0.53) & -0.025\end{array}$

$\begin{array}{llllllllllllllll}1,823 & 0.126(0.69) & 874 & 0.270(0.69) & 949 & -0.007 & 0.019(0.55) & 0.055(0.57) & -0.015 & 0.025(0.63) & 0.055(0.66) & -0.002 & 0.063(0.54) & 0.114(0.58) & 0.016(0.49)\end{array}$

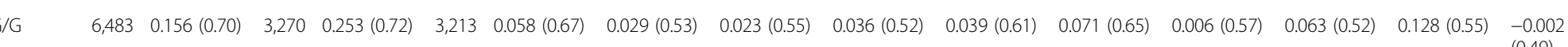

$P$ value $^{b}$

0.545

0.879

0.288

0.444

0.856

0.115

0.957

0.802

0.521

0.632

0.715

0.646 
Table 3 Association between genetic factors for neurodevelopmental problems (NDPs) and five SNPs (Continued)

\begin{tabular}{|c|c|c|c|c|c|c|c|c|c|c|c|c|c|c|c|c|}
\hline \multicolumn{3}{|c|}{ Effect size $(95 \% \mathrm{CI})^{\mathrm{C}}$} & \multicolumn{2}{|l|}{$\begin{array}{l}-0.010 \\
(-0.041 \\
0.022)\end{array}$} & \multicolumn{2}{|l|}{$\begin{array}{l}0.003 \\
(-0.04, \\
0.047)\end{array}$} & $\begin{array}{l}-0.026 \\
(-0.073, \\
0.022)\end{array}$ & $\begin{array}{l}-0.009 \\
(-0.033 \\
0.014)\end{array}$ & $\begin{array}{l}0.003 \\
(-0.029, \\
0.035)\end{array}$ & $\begin{array}{l}-0.030 \\
(-0.067 \\
0.007)\end{array}$ & $\begin{array}{l}0.001 \\
(-0.023, \\
0.024)\end{array}$ & $\begin{array}{l}-0.004 \\
(-0.033, \\
0.026)\end{array}$ & $\begin{array}{l}0.014 \\
(-0.029 \\
0.056)\end{array}$ & $\begin{array}{l}0.005 \\
(-0.016, \\
0.026)\end{array}$ & $\begin{array}{l}0.006 \\
(-0.025 \\
0.036)\end{array}$ & $\begin{array}{l}0.008 \\
(-0.026, \\
0.042)\end{array}$ \\
\hline \multicolumn{17}{|l|}{ SHANK3 } \\
\hline \multirow[t]{3}{*}{ rs9616915 0.49} & $\mathrm{C} / \mathrm{C}$ & 2,170 & $0.149(0.71)$ & 1,123 & $0.263(0.73)$ & 1,047 & $0.028(0.65)$ & $0.039(0.54)$ & $0.041(0.56)$ & $0.037(0.52)$ & $0.020(0.60)$ & $0.039(0.62)$ & $0.001(0.58)$ & $0.060(0.54)$ & $0.140(0.55)$ & $\begin{array}{l}-0.025 \\
(0.51)\end{array}$ \\
\hline & $\mathrm{C} / \mathrm{T}$ & 4,182 & $0.161(0.70)$ & 2,044 & $0.266(0.72)$ & 2,138 & $0.060(0.66)$ & $0.037(0.54)$ & $0.053(0.55)$ & $0.022(0.53)$ & $0.039(0.61)$ & $0.075(0.64)$ & $0.004(0.58)$ & $0.057(0.52)$ & $0.096(0.54)$ & $0.020(0.49)$ \\
\hline & $\mathrm{T} / \mathrm{T}$ & 2,061 & $0.120(0.69)$ & 1,048 & $0.232(0.70)$ & 1,013 & $0.005(0.66)$ & $\begin{array}{l}-0.010 \\
(0.52)\end{array}$ & $\begin{array}{l}-0.030 \\
(0.54)\end{array}$ & $0.011(0.50)$ & $0.042(0.63)$ & $0.083(0.68)$ & $\begin{array}{l}-0.001 \\
(0.57)\end{array}$ & $0.081(0.53)$ & $0.169(0.58)$ & $\begin{array}{l}-0.009 \\
(0.46)\end{array}$ \\
\hline \multicolumn{3}{|l|}{$P$ value $^{b}$} & 0.703 & & 0.870 & & 0.716 & 0.152 & 0.068 & 1.00 & 0.377 & 0.308 & 0.994 & 0.273 & 0.266 & 0.659 \\
\hline \multicolumn{3}{|c|}{ Effect size $(C \mid 95 \%)^{c}$} & $\begin{array}{l}0.004 \\
(-0.017 \\
0.025)\end{array}$ & & $\begin{array}{l}0.002 \\
(-0.027 \\
.032)\end{array}$ & & $\begin{array}{l}0.006 \\
(-0.025 \\
0.037)\end{array}$ & $\begin{array}{l}-0.012 \\
(-0.028 \\
0.004)\end{array}$ & $\begin{array}{l}-0.020 \\
(-0.042 \\
0.001)\end{array}$ & $\begin{array}{l}0.001 \\
(-0.025 \\
0.025)\end{array}$ & $\begin{array}{l}0.007 \\
(-0.009, \\
0.023)\end{array}$ & $\begin{array}{l}0.011 \\
(-0.01 \\
0.031)\end{array}$ & $\begin{array}{l}0.001 \\
(-0.028 \\
0.028)\end{array}$ & $\begin{array}{l}0.008 \\
(-0.006 \\
0.022)\end{array}$ & $\begin{array}{l}0.012 \\
(-0.009, \\
0.032)\end{array}$ & $\begin{array}{l}0.005 \\
(-0.017 \\
0.028)\end{array}$ \\
\hline
\end{tabular}

${ }^{\mathrm{a}}$ Mean values (SD).

bUncorrected $P$ value.
${ }^{C}$ Effect size presented as the regression coefficient $(95 \% \mathrm{CI})$.

A-TAC: Autism-Tics, ADHD, and Other Co-morbidities inventory. 
influence of common variation in SHANK3 or RELN on autistic-like traits, which has not been investigated previously. However, in our study we did not find significant associations between ALTs and rs9616915 or rs362691 (Table 1).

Since there is a large co-morbidity between NDPs, we also explored the influence of our ASD candidate SNPs on four genetic NDP factors designed to have $100 \%$ heritability [18], that is, we explored the genetic etiology of the co-morbidity among NDPs. These analyses did not show a major influence of these SNPs on the genetic NDP factors (Table 3).

In line with the GWAS published for autistic traits $[22,23]$, we did not find significant association between the investigated SNPs and ALTs in our large population, although nominal significance is observed for the CNTNAP2 SNP rs7794745. Hence, our results suggest that these genes, which previously have been found associated with ASD diagnosis, do not have any major influence on ALTs in children from the general population. However, it cannot be excluded that other variants in the same genes may have an effect since our selected SNPs do not capture all variations within our selected genes. Further studies that investigate the influence of genetic variation on different measures of ALTs are highly warranted to better understand the genetics of autism related phenotypes and neurodevelopmental disorders.

\section{Conclusion}

Taken together, our association analyses between the investigated autism candidate regions and ALTs do not suggest a major influence on ALTs in children from the general population.

\section{Additional file}

Additional file 1: Association analyses adjusted for age. The additional file includes three additional Tables. Table S1. Association analyses between autistic-like traits and five SNPs after adjustment for age. Table S2. ASD case-control analyses after adjustment for age and Table S3. Association analyses between genetic factors for neurodevelopmental problems and five SNPs after adjustment for age.

\section{Abbreviations \\ A-TAC: Autism-Tics, Attention-Deficit/Hyperactivity Disorder, and Other Co- morbidities inventory; ALT: Autistic-like trait; ASD: Autism Spectrum disorder; CATSS: Child and Adolescent Twin Study in Sweden; \\ CNTNAP2: Contactin associated protein-like 2; NDP: Neurodevelopmental Problem; RELN: Reelin; SHANK3: SH3 and multiple ankyrin repeat domains 3; SNP: Single nucleotide polymorphism.}

\section{Competing interests}

The authors declare that they have no competing interests.

\section{Authors' contributions}

$J M$ and $L$ conceived of the study and designed the experiments in collaboration with LW and PL. LJ carried out the laboratory work, performed statistical analyses, and was responsible for the handling of samples for genotyping. LW provided financial support, participated in the statistical analyses, and performed results interpretations. DH and AZ participated in the statistical analyzes, acquisition of data, and performed results interpretations. HA developed the A-TAC questionnaire and interpreted the A-TAC data together with SL. EP developed and interpreted the results on the genetic factor for neurodevelopment problems. PL, HA, and SL are responsible for the recruitment of subjects of the CATSS population. All authors made critical revision of the manuscript. JM provided financial support and wrote the manuscript together with $L$. All authors read and approved the final manuscript.

\section{Acknowledgements}

We are grateful to the study participants and their relatives who have made this study possible. This work has been supported by the Swedish Research Council, the Swedish Council for Working Life and Social Research, The Petrus and Augusta Hedlund Foundation, Åke Wiberg foundation, Åhlens Foundation, Wilhelm and Martina Lundgren Foundation, and the Sahlgrenska Academy.

\section{Author details}

'Department of Pharmacology, Institute of Neuroscience and Physiology at the Sahlgrenska Academy, University of Gothenburg, POB 431, SE 405 30, Gothenburg, Sweden. ${ }^{2}$ Department of Medical Epidemiology and Biostatistics, Karolinska Institutet, Stockholm, Sweden. ${ }^{3}$ Department of Forensic Psychiatry, Institute of Neuroscience and Physiology at the Sahlgrenska Academy, University of Gothenburg, Gothenburg, Sweden. ${ }^{4}$ Swedish Prison and probation service, R\&D unit, Gothenburg, Sweden. ${ }^{5}$ Gillberg Neuropsychiatry Centre, Institute of Neuroscience and Physiology at the Sahlgrenska Academy, University of Gothenburg, Gothenburg, Sweden.

Received: 19 June 2014 Accepted: 20 November 2014

Published: 16 December 2014

\section{References}

1. Leitner $Y$ : The co-occurrence of autism and attention deficit hyperactivity disorder in children - what do we dnow? Front Hum Neurosci 2014, 8:268.

2. Lichtenstein P, Carlstrom E, Rastam M, Gillberg C, Anckarsater H: The genetics of autism spectrum disorders and related neuropsychiatric disorders in childhood. Am J Psychiatry 2010, 167:1357-1363.

3. State MW, Levitt $P$ : The conundrums of understanding genetic risks for autism spectrum disorders. Nat Neurosci 2011, 14:1499-1506.

4. Weiss LA, Arking DE, Daly MJ, Chakravarti A: A genome-wide linkage and association scan reveals novel loci for autism. Nature 2009, 461:802-808.

5. Wang K, Zhang H, Ma D, Bucan M, Glessner JT, Abrahams BS, Salyakina D, Imielinski M, Bradfield JP, Sleiman PM, Kim CE, Hou C, Frackelton E, Chiavacci R, Takahashi N, Sakurai T, Rappaport E, Lajonchere CM, Munson J, Estes A, Korvatska O, Piven J, Sonnenblick LI, Alvarez Retuerto Al, Herman El, Dong H, Hutman T, Sigman M, Ozonoff S, Klin A: Common genetic variants on 5 p14.1 associate with autism spectrum disorders. Nature 2009, 459:528-533.

6. Anney R, Klei L, Pinto D, Regan R, Conroy J, Magalhaes TR, Correia C, Abrahams BS, Sykes N, Pagnamenta AT, Almeida J, Bacchelli E, Bailey AJ, Baird G, Battaglia A, Berney T, Bolshakova N, Bolte S, Bolton PF, Bourgeron T, Brennan S, Brian J, Carson AR, Casallo G, Casey J, Chu SH, Cochrane L, Corsello C, Crawford EL, Crossett A: A genome-wide scan for common alleles affecting risk for autism. Hum Mol Genet 2010, 19:4072-4082.

7. Lundstrom S, Chang Z, Rastam M, Gillberg C, Larsson H, Anckarsater H, Lichtenstein P: Autism spectrum disorders and autisticlike traits similar etiology in the extreme end and the normal variation. Arch Gen Psychiat 2012, 69:46-52.

8. Ronald A, Happe F, Bolton P, Butcher LM, Price TS, Wheelwright S, Baron-Cohen S, Plomin R: Genetic heterogeneity between the three components of the autism spectrum: a twin study. J Am Acad Child Adolesc Psychiatry 2006, 45:691-699.

9. Pourcain BS, Wang K, Glessner JT, Golding J, Steer C, Ring SM, Skuse DH, Grant SFA, Hakonarson H, Smith GD: Association between a high-risk autism locus on $5 \mathrm{p} 14$ and social communication spectrum phenotypes in the general population. Am J Psychiat 2010, 167:1364-1372. 
10. Whitehouse AJ, Bishop DV, Ang QW, Pennell CE, Fisher SE: CNTNAP2 variants affect early language development in the general population. Genes Brain Behav 2011, 10:451-456.

11. Alarcon M, Abrahams BS, Stone JL, Duvall JA, Perederiy JV, Bomar JM, Sebat J, Wigler M, Martin CL, Ledbetter DH, Nelson SF, Cantor RM, Geschwind DH: Linkage, association, and gene-expression analyses identify CNTNAP2 as an autism-susceptibility gene. Am J Hum Genet 2008, 82:150-159.

12. Strauss KA, Puffenberger EG, Huentelman MJ, Gottlieb S, Dobrin SE, Parod JM, Stephan DA, Morton DH: Recessive symptomatic focal epilepsy and mutant contactin-associated protein-like 2. N Engl J Med 2006, 354:1370-1377.

13. Persico AM, D'Agruma L, Maiorano N, Totaro A, Militerni R, Bravaccio C, Wassink TH, Schneider C, Melmed R, Trillo S, Montecchi F, Palermo M, Pascucci T, Puglisi-Allegra S, Reichelt KL, Conciatori M, Marino R, Quattrocchi CC, Baldi A, Zelante L, Gasparini P, Keller F: Reelin gene alleles and haplotypes as a factor predisposing to autistic disorder. Mol Psychiatry 2001, 6:150-159.

14. Durand CM, Betancur C, Boeckers TM, Bockmann J, Chaste P, Fauchereau F, Nygren G, Rastam M, Gillberg IC, Anckarsater H, Sponheim E, GoubranBotros H, Delorme R, Chabane N, Mouren-Simeoni MC, de Mas P, Bieth E, Roge B, Heron D, Burglen L, Gillberg C, Leboyer M, Bourgeron T: Mutations in the gene encoding the synaptic scaffolding protein SHANK3 are associated with autism spectrum disorders. Nat Genet 2007, 39:25-27.

15. Anckarsater H, Lundstrom S, Kollberg L, Kerekes N, Palm C, Carlstrom E, Langstrom N, Magnusson PKE, Halldner L, Bolte S, Gillberg C, Gumpert C, Rastam M, Lichtenstein P: The Child and Adolescent Twin Study in Sweden (CATSS). Twin Res Hum Genet 2011, 14:495-508.

16. Hansson SL, Svanstrom Rojvall A, Rastam M, Gillberg C, Anckarsater H: Psychiatric telephone interview with parents for screening of childhood autism - tics, attention-deficit hyperactivity disorder and other comorbidities (A-TAC): preliminary reliability and validity. Br J Psychiatry 2005, 187:262-267.

17. Larson T, Anckarsater H, Gillberg C, Stahlberg O, Carlstrom E, Kadesjo B, Rastam M, Lichtenstein P: The autism-tics, AD/HD and other comorbidities inventory (A-TAC): further validation of a telephone interview for epidemiological research. BMC Psychiatry 2010, 10:1

18. Pettersson $\mathrm{E}$, Anckarsater $\mathrm{H}$, Gillberg C, Lichtenstein P: Different neurodevelopmental symptoms have a common genetic etiology. J Child Psychol Psychiatry 2013, 54:1356-1365.

19. Gauderman WJ: Sample size requirements for association studies of gene-gene interaction. Am J Epidemiol 2002, 155:478-484.

20. Arking DE, Cutler DJ, Brune CW, Teslovich TM, West K, lkeda M, Rea A, Guy $M, L i n S$, Cook EH, Chakravarti A: A common genetic variant in the neurexin superfamily member CNTNAP2 increases familial risk of autism. Am J Hum Genet 2008, 82:160-164.

21. Ma D, Salyakina D, Jaworski JM, Konidari I, Whitehead PL, Andersen AN, Hoffman JD, Slifer SH, Hedges DJ, Cukier HN, Griswold AJ, McCauley JL, Beecham GW, Wright HH, Abramson RK, Martin ER, Hussman JP, Gilbert JR, Cuccaro ML, Haines JL, Pericak-Vance MA: A genome-wide association study of autism reveals a common novel risk locus at 5p14.1. Ann Hum Genet 2009, 73:263-273.

22. Jones RM, Cadby G, Melton PE, Abraham L, Whitehouse AJ, Moses EK: Genome-wide association study of autistic-like traits in a general population study of young adults. Front Hum Neurosci 2013, 7:658

23. Ronald A, Butcher LM, Docherty S, Davis OS, Schalkwyk LC, Craig IW, Plomin $R:$ A genome-wide association study of social and non-social autistic-like traits in the general population using pooled DNA, $500 \mathrm{~K} \mathrm{SNP}$ microarrays and both community and diagnosed autism replication samples. Behav Genet 2010, 40:31-45.

24. Wang Z, Hong Y, Zou L, Zhong R, Zhu B, Shen N, Chen W, Lou J, Ke J, Zhang $T$, Wang W, Miao $X$ : Reelin gene variants and risk of autism spectrum disorders: an integrated meta-analysis. Am J Med Genet B Neuropsychiatr Genet 2014, 165:192-200.

25. Serajee FJ, Zhong H, Mahbubul Huq AH: Association of Reelin gene polymorphisms with autism. Genomics 2006, 87:75-83.

26. Qin J, Jia M, Wang L, Lu T, Ruan Y, Liu J, Guo Y, Zhang J, Yang X, Yue W, Zhang D: Association study of SHANK3 gene polymorphisms with autism in Chinese Han population. BMC Med Genet 2009, 10:61.

doi:10.1186/2040-2392-5-55

Cite this article as: Jonsson et al:: Association study between autistic-like traits and polymorphisms in the autism candidate regions RELN CNTNAP2, SHANK3, and CDH9/10. Molecular Autism 2014 5:55.

\section{Submit your next manuscript to BioMed Central and take full advantage of:}

- Convenient online submission

- Thorough peer review

- No space constraints or color figure charges

- Immediate publication on acceptance

- Inclusion in PubMed, CAS, Scopus and Google Scholar

- Research which is freely available for redistribution

Submit your manuscript at www.biomedcentral.com/submit
Ciomed Central 